\title{
Dispositional Humility Of Clinicians In An Interprofessional Primary Care Environment: A Mixed Methods Study
}

This article was published in the following Dove Press journal: Journal of Multidisciplinary Healthcare

\section{Masa Sasagawa (iD \\ Paul S Amieux (D) \\ Bastyr University Research Institute, Kenmore, WA 98028, USA}

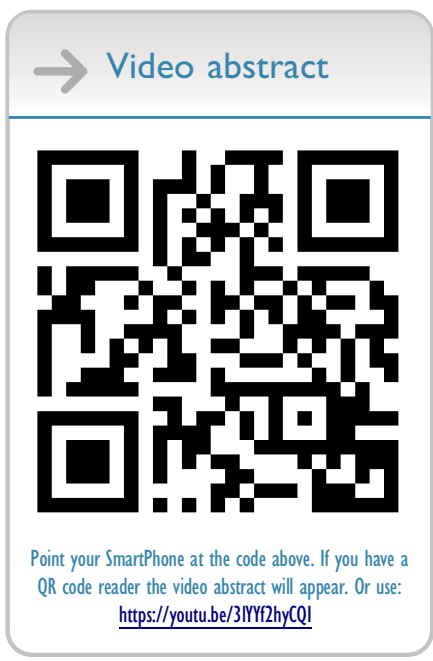

Correspondence: Masa Sasagawa Bastyr University Research Institute, NE, Kenmore, WA 98028, USA

Email msasagawa@bastyr.edu
Objectives: Dispositional humility in professionals is a character trait that allows one to monitor self-centered occupational drive and to pay attention to the needs of other professionals. The aim of this study is to test whether or not clinicians working in interprofessional team care environments identify the character trait of humility as an important factor for successful collaborative relationships. This study aimed to revise a concept map of dispositional humility created through literature review.

Design: An explanatory sequential mixed-methods study was composed of the HEXACO personality test and the Integrative Medicine Attitude Questionnaire, followed by summative and directed content analyses of one-on-one interview data in order to identify the element of dispositional humility.

Setting: In the State of Washington, USA, where physicians (MD/DO), nurse practitioners (NP) and naturopathic clinicians (ND) serve Medicaid patients in community clinics.

Participants: 6 MDs, 4 NPs, and 11 NDs.

Results: Twenty-one primary care clinicians were enrolled. Fifteen clinicians completed the interview. It was observed that the: 1) honesty-humility trait $(\mathrm{p}<0.01)$, conscientiousness $(\& p<0.01)$, and openness to experience $(p<0.05)$ domains of primary care clinicians were statistically significantly higher than the reference standards; 2 ) attitudes toward integrative medicine did not differentiate the different clinician types; and 3) qualitative data supported the component of dispositional humility as a desirable trait in professionals with whom they would like to work.

Conclusion: To maintain high-quality patient care while working as a team, limiting selfinterest while focusing on the needs of others may be necessary and in the best interest of patients. An attitude of accepting the principles of integrative medicine has permeated this sample of primary healthcare workers. Both quantitative and qualitative analyses revealed that humility was viewed as an important character trait for successful interprofessional collaboration. A revised concept map of dispositional humility to enhance collaborative relationships was created.

Keywords: primary health care, interprofessional relations, interdisciplinary placement, dispositional humility, collaboration

\section{Introduction}

This study posed the question of whether or not clinicians perceive humility as an important factor for interprofessional collaboration in an integrative primary care setting. Since the early 1990s, US healthcare consumers have spent an increasing amount of healthcare dollars for non-conventional complementary and alternative medicine. ${ }^{4}$ As a result of this demand, over the past two decades, conventional 
healthcare systems have been adjusting and responding to this desire on the part of healthcare consumers. "Integrative Medicine" is a term used to describe such combined care in the conventional setting. As an example of this, the implementation of multidisciplinary professional team care was found effective in managing knee osteoarthritis in a primary care setting; ${ }^{5}$ however, power dynamics and mistrust among clinicians with different professional healthcare training and credentials can make professional interactions challenging. ${ }^{6}$ To maintain patient-centered professional teamwork, limiting selfinterest and focusing on the needs of other professionals may prove essential; furthermore, "being too arrogant or self-focused could negatively affect relationship quality". 8 Skills building such as effective communication skills in primary care increased health outcomes. ${ }^{9}$ This study explored the role of a personality factor, dispositional humility, in enhancing the performance and function of integrative healthcare teams.

Dispositional humility is defined as a native capacity for self-reflection and the ability to achieve a balance between self-interest and the needs of others. ${ }^{1}$ Primary care clinicians who serve patients in community health clinics may possess certain job-related characteristics like altruism. One of the assumptions of this study was that clinicians who choose to serve underprivileged populations (those on Medicaid, for example) may naturally possess dispositional factors that foster positive and productive relationships not only with patients but also with professional colleagues. More specifically, people who elect to work in challenging healthcare work environments and still fulfill their obligations may be associated with the character trait of humility. Nonetheless, one-third of office-based physicians in the US did not accept new Medicaid-insured patients in 2011-2012, ${ }^{10}$ and although fee increases would be predicted to change this low enrollment rate, fee increases did not change the participation rate in 2018. ${ }^{11}$ The Association of Family Medicine Residency Directors (AFMRD) Board, as articulated by Mark Robinson et al (2008), states that: "Faculty, staff, and residents spend many hours carefully scrutinizing dean's letters, transcripts, USMLE scores, letters of recommendation and interview data" for the National Match process for residency positions. The AFMRD Board looks for character traits consistent with what one would expect in a good family physician, traits that include being trustworthy, loyal, helpful, friendly, courteous, kind, obedient, cheerful, thrifty, brave, clean and reverent. ${ }^{12}$ Many of the character traits described above can be associated with the character trait of humility; however, assessing the level of humility of individuals can be complex. Truly humble individuals may not proclaim themselves to be humble. Therefore, in order to study humility, along with validated self-reported survey results, other methods to assess humility are needed. This study elected to use a mixed-methods approach by first having clinicians selfevaluate a personality trait followed by interviews to identify personality and environmental factors that facilitate or interfere with collaboration. If components of humility emerged from the interview data as personality or environmental factors viewed as important for collaboration, the data would confirm the perceived importance of dispositional humility.

\section{Methods}

\section{Patient And Public Involvement}

This study investigated the clinician's characteristics and environment conducive to collaboration. Patients and the public were not involved in recruitment or in the study. The results from this study will be most useful if disseminated to health professional school administrators and clinician recruiters. The targeted population for recruitment was primary care clinicians serving Medicaid patients in Seattle, Washington, USA. These clinicians had healthcare professional training backgrounds and credentials in medicine, nursing, or naturopathic medicine. Medicaid-serving clinics were chosen for two reasons: 1) to seek clinicians with dispositional humility that might be demonstrated by working in such inherently stressful and demanding healthcare environments; and 2) naturopathic doctors are licensed and equivalently able to serve Medicaid patients as primary care providers in the State of Washington. MD/DOs were board-certified family medicine physicians or internists; nurse practitioners (NPs) were master's level ARNPs; and naturopathic doctors (NDs) had two to 3 years of residency training after the completion of the doctoral degree. The diagrammatic process of this study design is depicted in Supplement A: Research Design.

\section{Quantitative Data}

The 60-item HEXACO personality inventory ${ }^{2}$ is a validated dispositional assessment tool measuring the six domains of personality: Honesty-Humility, Emotionality, eXtraversion, Agreeableness, Conscientiousness, and Openness to 
experience, and one of the six, the Honest-Humility (HH) domain, was found to have four facets (subdomains): sincerity, fairness, greed-avoidance, and modesty. ${ }^{13}$ A priori power calculation was based on the $\mathrm{HH}$ domain with a reference mean and standard deviation of 3.23 and 0.66 , respectively. ${ }^{2}$ By assuming the same level of standard deviation and $15 \%$ difference between the mean of the sample and the reference point, a sample size of 14 was necessary to obtain a power of 0.8 with an alpha level of 0.05 . The 29-item Integrative Medicine Attitude Questionnaire (IMAQ) ${ }^{3}$ quantifies two factors: 1) openness to new ideas and paradigms; and 2) value of both introspection and relationship with patients. Patients' preferences for integrative medicine have provided the US healthcare system with opportunities for clinicians to work with other clinical professionals with different training and health perspectives. Primary care clinicians in this study were divided into conventionally trained clinicians (MD, DO, or NP) and complementary and alternative medicine (CAM) trained naturopathic doctors (ND). In a 2005 publication, IMAQ scores of conventionally trained clinicians and CAM clinicians were different by a $\mathrm{t}$-score of $12.05(\mathrm{df}=191)$. We calculated a sample size of 7 would be sufficient to achieve a statistical power of 0.8 at an alpha level of 0.05 between conventionally trained clinicians versus NDs who were trained from the perspective of CAM. Survey data were collected in the Research Electronic Data Capture (REDCap) system. ${ }^{14}$ REDCap is an application developed by Vanderbilt University and collects virtually any type of data in a 21 CFR Part 11, FISMA, and HIPAA-compliant environment. The data in REDCap were downloaded and analyzed using SPSS version $25^{15}$ and GraphPad Prism version 7.01 for Windows.

\section{Qualitative Data}

The surveyed participants were invited to participate in a brief semi-structured interview at the end of survey and were able to leave their contact information. The interview was conducted in-person or by telephone. The recorded interview responses were transcribed verbatim and loaded into HyperResearch (v3.5.2) software for coding and summative data analysis. Representative samples (balanced sampling of 2 MDs, 2 NPs, and 2 NDs) from the interviews were used for the directed content analysis. The directed content analysis was based on a published concept map paper entitled: "Concept analysis of dispositional humility among professionals in an interdisciplinary healthcare environment." (Figure 1)

Hsieh and Shannon (2005) describe three types of content analyses: conventional, summative, and directed content analysis. ${ }^{7}$ Conventional content analysis usually does not start from preconceived coding expectations, and lets themes emerge as the content is interpreted and coded. Summative content analysis relies on the frequency statistics of abstracted codes. Directed content analysis uses a preconceived model and looks for codes related to the model; hence the coding is directed. ${ }^{7}$ The coding, categorization and thematic extraction of the interview data were matched to the concept map already created. ${ }^{1}$ Finally, based on the results from the summative and directed content data analyses, the concept map was refined. The original study design and protocol modification were approved by the Bastyr University Institutional Review Board (IRB: 16-1558). All datasets used and/or analyzed during the current study are available from the corresponding author upon reasonable request for 7 years from the date of publication.

\section{Results}

During 2016-17, 32 people accessed the survey and 21 clinicians consented online and completed the survey, and 14 of 21 of the survey participants consented by written informed consent for the interview. One additional clinician provided written consent directly for the interview without taking the survey, giving a total of 15 interviews. The flow chart of recruitment is diagrammed in Figure 2.

A total of 15 individuals completed the interview (five out of six MDs, two out of four NPs, eight out of eleven NDs) and a total of 21 individuals completed the survey. Their median years of practice were 7.0 (MDs), 8.5 (NPs), 10.0 (NDs) with an overall median of 7.0. Three missing values were filled by using the calculated mean value.

\section{HEXACO}

Six multiple independent t-tests were conducted for each domain of HEXACO between the mean of the clinician's score and the reference standard. The significance level was adjusted using the Holm-Sidak method for multiple comparisons to avoid alpha error. ${ }^{16}$ Clinicians from this sample showed higher Honesty/Humility, Conscientiousness, and Openness domains ( $\mathrm{n}=21)$. The $\mathrm{Z}$-scores were used to depict the relative difference between the clinician's dispositional factors relative to the reference values, which form a perfect hexagon (yellow line). (Figure 3)

The combination of high $\mathrm{HH}$ and Conscientiousness describes a character that is more resilient against work stressors, able to behave conscientiously under stressful conditions, and maintain truthful and non-deceptive 


\section{Concept Map of Dispositional Humility}

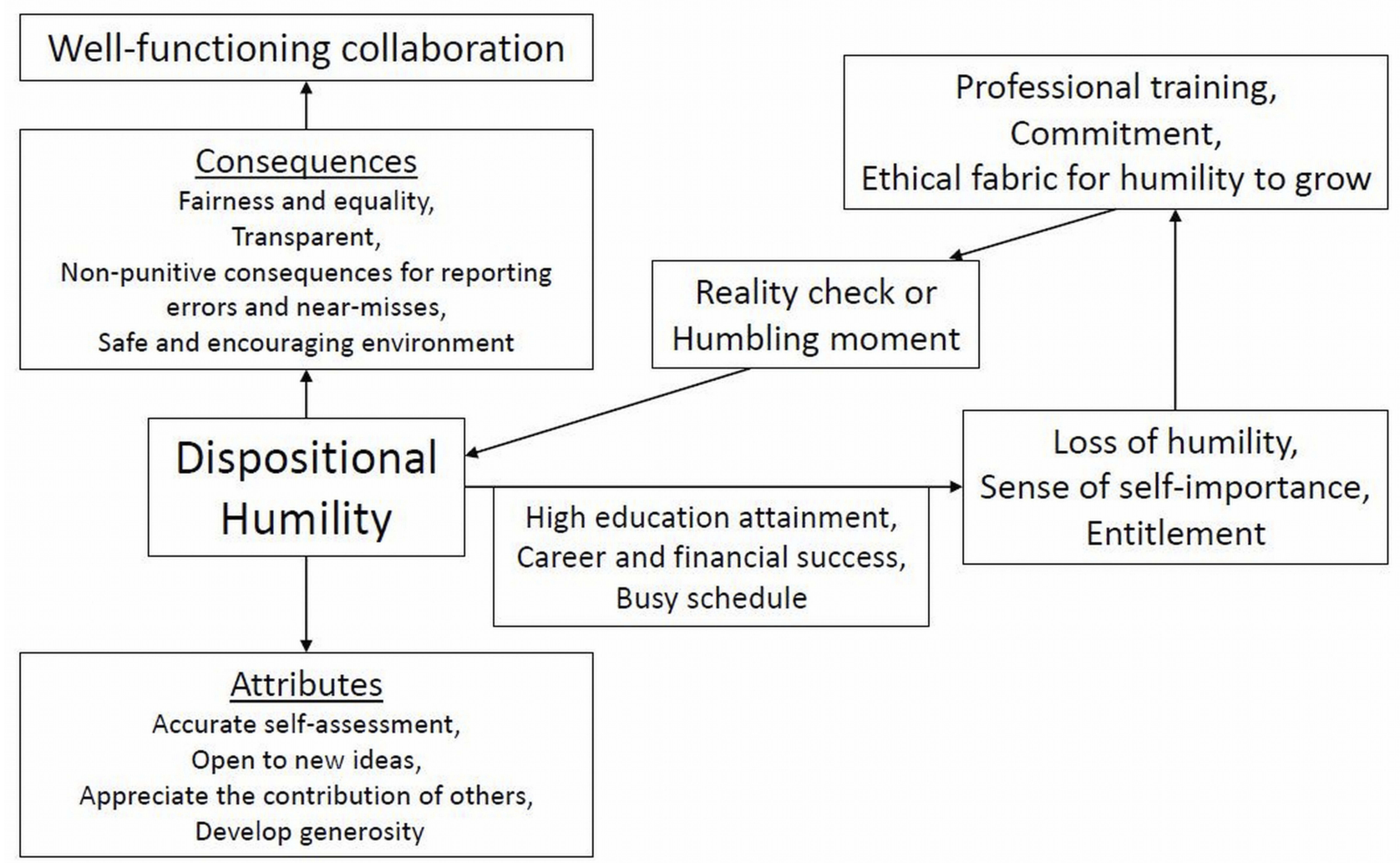

Figure I Dispositional humility allows clinicians to have an accurate self-assessment, be open to new ideas, appreciate the contribution of others, and develop generosity. Dispositional humility in leaders can facilitate character development of team members and create an environment characterized by fairness and equality, transparency, nonpunitive consequences for reporting errors and near-misses, and a safe and encouraging environment for performing work. However, dispositional humility must be nurtured and developed through professional training because high educational attainment, career and financial success, and busy schedules may lead to a sense of self-importance and entitlement that can promote separation of team members into hierarchies based on professional disciplines and specialties. Note: this is not structural equation modeling, and the pathways or factors were not statistically analyzed. Copyright (C) 2019. Dove Medical Press. Reproduced from Sasagawa M, Amieux P. Concept map of dispositional humility among professionals in an interdisciplinary healthcare environment: Qualitative synthesis. J Multidisciplinary Healthcare. 2019;12:543-554.'

relationships. It may also indicate the tendency to trust others as well as to become trustworthy individuals themselves. The openness domain indicates the ability to accept new ideas and criticism from others. By combining these characteristics, these clinicians are consistent with the attributes and consequences of having dispositional humility.

\section{IMAQ}

The twenty-one participants were divided into 10 conventional clinicians (MD, NP) and 11 complementary and alternative or non-conventional clinicians (ND). The t-tests showed that the clinicians with different professional healthcare training and credentials were not statistically different for the two subdomains: 1) "openness to new ideas and paradigm" $\mathrm{t}(19)=1.128, \mathrm{p}=0.472$; and 2) "value of both introspection and relationship with patients" $t(19)=0.272, p=0.955$. From an integrative medicine perspective, non-conventionally trained ND clinicians were not different from conventionally trained medical and nursing clinicians, or perhaps the conventional medical and nursing training had incorporated the perspective of integrative medicine in the past decade. ${ }^{3}$

\section{Qualitative Data}

We avoided any suggestion of studying the character trait of humility as much as possible during recruitment, informed consent process, and interview. "Humility" and conceptually related terms such as "arrogant" or "humble" were not used during conversations unless they were mentioned by participants. The focus of discussion was to collect information regarding personality and environmental factors that enhance or interfere with collaborative work in interdisciplinary primary healthcare clinics and 


\section{Recruitment}

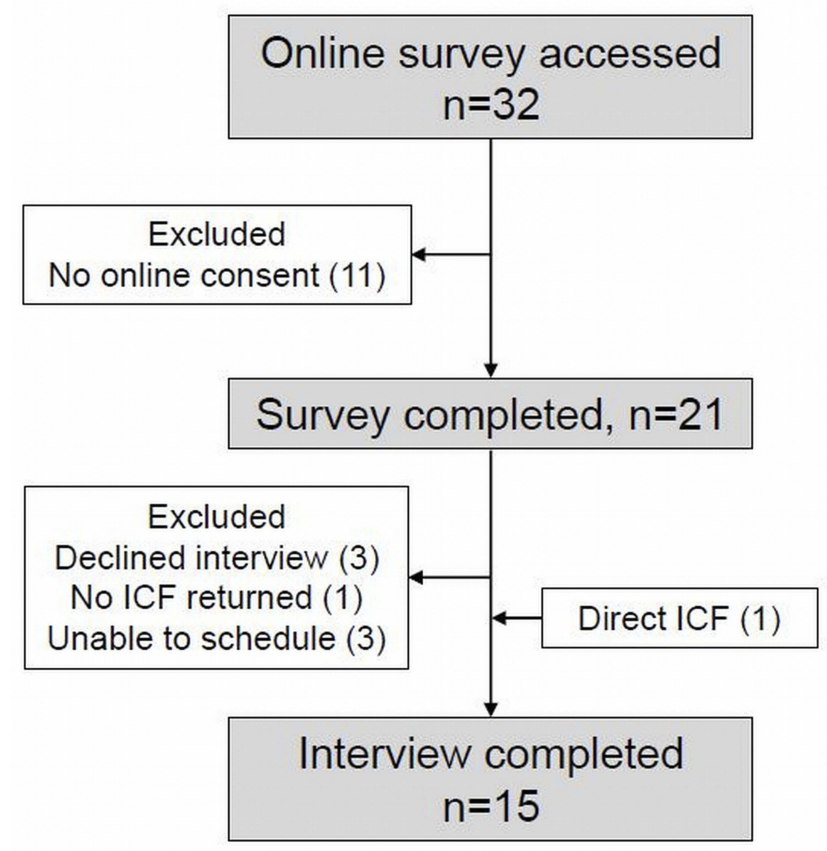

Figure 2 Shows the overall participation in the two phases of data collection. One MD directly contacted the author for interview participation without completing the online survey. Per protocol, this interview data were included.

centers. Authors familiarized themselves with the transcript before coding. Based on the interview question, codes were categorized into: 1) negative dispositional factors; 2) positive dispositional factors; 3 ) positive environmental factors; and 4) negative environmental factors. Coding was repeated iteratively to achieve interpretive saturation. The HyperResearch (v3.5.2) software organized the codes and created the summative statistics. The expressions seem diverse due to the nature of asking openended questions; however, some of the expressions were coherent with the attributes and consequences of having dispositional humility, as described by Figure 1. An excerpt of the summative content data is shown in Supplement B: Summative Codes.

Below is a list of bullet points of the descriptors of humility extracted by two investigators.

Humility components that emerged as a desirable factor by clinicians

- Friendly, open, and approachable

- Patient-centered (other-oriented and professional duty-orientated)

- Humility

- Trust

\section{Z-score HEXACO profile of all PCP $(n=21)$}

-PCP ( $n=21)$ Reference $(\mathrm{n}=1,126)$
Openness
( $\mathrm{p}=0.019)$

Figure 3 To illustrate the overall characteristics, each clinician's HEXACO score was converted to the Z-score based on the reference values. The clinicians showed statistically higher Honesty/Humility, Openness, and Conscientiousness domains compared to the reference group of undergraduate college samples. $(n=I, 126)$. *Statistical significance at the alpha level 0.05 . $* *$ Statistical significance at the alpha level 0.01 .

- Modest self-portrayal (accurate self-assessment)

- Be responsible/respectful of other people's time

Dispositional factors that emerged as a barrier, making the person hard to work with

- Egotistical

- Arrogance

- Closed-minded

- Dogmatic, self-interest medicine (not evidence-based)

- Questionable value (colleagues who hold non-evidence-based beliefs about particular modalities)

Helpful factor/environment for collaboration

- Opportunity to develop trust and collegial relationships

- Open common space to communicate, not separate rooms

- Increase knowledge of other professional colleagues

- Warm handoffs and fact-to-face interactions

- Developing a culture of mutual respect 
- Having a Reality Check

Environmental or situational hindrance for collaboration

- Unfairness (perceived or real)

- Lack of resources

- Knowledge gap about other professionals

\section{Directed Content Analysis}

Six representative interviews out of fifteen were analyzed by two authors independently. Directed content analysis looked for the components of factors associated with the preconceived model (Figure 1).

Themes to improve/revise Figure 1

\section{Busy Schedule And Lack Of Time}

This theme was consistent with previously published data on primary care clinicians. ${ }^{17}$ Similar expressions were found within statements such as requesting more administrative time, spending non-working time with colleagues (to get to know each other), having bad timing for a warm handoff, not planning well, not having work organized, and not respecting the time of colleagues. The consequences of this theme were to convey the impression of arrogance and selfishness that was consistent with the model.

\section{Lack Of Humility Or Loss Of Humility}

Perceptions such as egotistical, arrogant, closed-minded, dogmatic, inflexible and over-confident could be the result of having busy interactions (above) or dispositional issues; however, lack of humility was confirmed to be a negative factor to collaborative work.

\section{Professional Training}

Clinicians were aware of the need for constant learning. Getting to know colleagues, including their specialties and capacities, was part of a desire to have a smooth working relationship. This was also consistent with the model; however, the interviewees conveyed the message that such learning opportunities did not have to be humiliating; it could be a form of social gathering. Thus, this part of the model was modified.

\section{Reality Check}

During professional training, rigorous training curricula and interaction with mentors, teachers and trainers with advanced skills and knowledge seemed to provide a reality check or humbling moment. This element of the model was confirmed by the interview data.

\section{Attributes Of Humility}

Many attributes were described in a negative manner such as egotistic, arrogant, self-absorbed, selfish, over-confident, inflexible, narrow or closed-minded, rather than a positive manner such as open to new ideas or openminded.

\section{Consequences}

An environment or opportunity to become vulnerable was brought up. This vulnerability may not be a direct consequence of dispositional humility but it signifies an environment in which professionals do not have to be afraid to trust and be trusted. In the model, this could be interpreted as a safe and encouraging environment for collaboration because you do not have to be afraid of coworkers who might react negatively to collaboration requests.

Figure 4 shows a modified version of the published concept map. The themes or dynamic elements that were not confirmed during the summative and directed content analyses are in grey scale.

Methods to increase the scientific rigor (design, data collection, analysis and drawing of conclusions) of this qualitative analysis are summarized in Table 1. The lefthand column indicates the components of scientific rigor discussed by previous researchers, and the right-hand column indicates the process used for this study.

\section{Discussion}

High $\mathrm{HH}$ indicates prosocial orientation and behaviors as well as the expectation that others will be the same and trustworthy. ${ }^{21}$ Because conscientiousness also reflects the level of ethical strength, ${ }^{22}$ by combining these components together we hypothesize that individuals with high $\mathrm{HH}$ and Conscientiousness scores will uphold ethical principles while developing trusting relationship with others. Nonetheless, high educational attainment, career and financial success, busy schedules and stressful environments may lead to a loss of humility, leading to a sense of self-importance, entitlement, and disregard for the needs of others. Professional training is not only competency-based skill building but in its best manifestations is also a life-long commitment to learning, reassessment and reappraisal of what defines professionalism, and the development of an ethical culture that promotes a sense of mutual respect and humility. Professionals who lose their 


\section{Concept Map of Dispositional Humility}

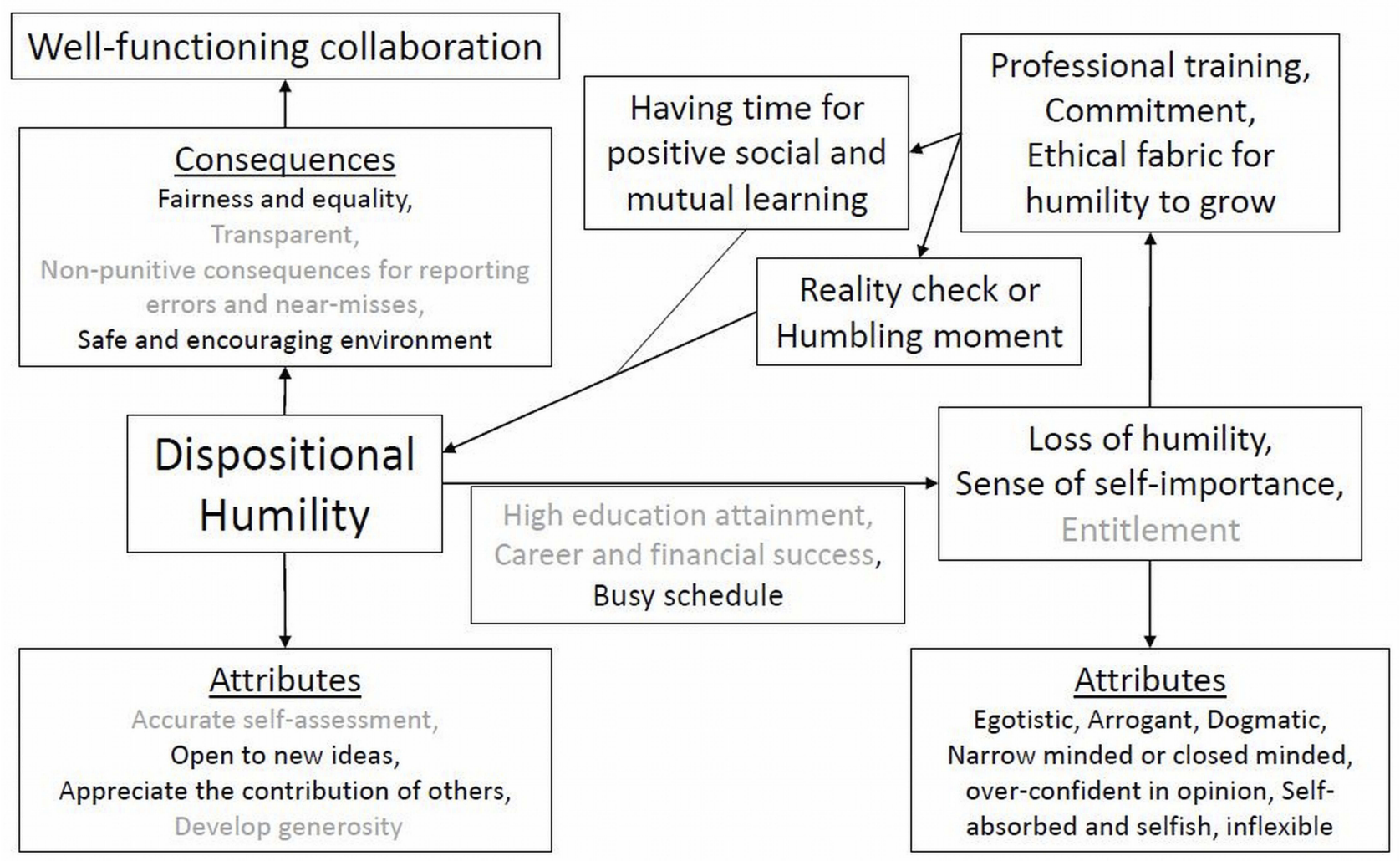

Figure $4 \mathrm{~A}$ proposed concept map diagram describing factors associated with dispositional humility and a possible mechanism of how these factors allow clinicians to enhance collaboration among professionals. This figure was made by revising Figure I of this article. The three gray scales indicate a new theme/category that appeared in this study (bold), a theme/category that was confirmed by the qualitative analysis (regular), and themes/categories which did not appear in the interview (gray scale).

sense of humility may face a reality check or humbling moment that awakens the sense of humility; however, how one faces and reacts to a reality check or humbling moment will depend upon the mental and emotional maturity of the individual. Retaliatory behavior and counter-attack against fair and constructive criticism may

Table I Scientific Rigor Of This Qualitative Analysis (Partly From Lincoln Y, guba ${ }^{18}$ )

\begin{tabular}{|c|c|}
\hline Rigor Component & Collection And Analysis Of Interview Data \\
\hline Credibility (internal validity) & $\begin{array}{l}\text { Obtain positive and negative data (easy/hard, facilitative/disruptive personality and environment), } \\
\text { empower participants with positive interactions during the interview, hiding the real agenda } \\
\text { (dispositional humility) in ethical ways (IRB approved per protocol) }\end{array}$ \\
\hline $\begin{array}{l}\text { Dependability (reliability, consistency or } \\
\text { stability of data) }\end{array}$ & $\begin{array}{l}\text { (weakness) short interview session ( } 20-30 \text { min interview time), attempt to weight the input from } \\
\text { different clinical credentials, expanding the subject of humility when it was brought up by participants, } \\
\text { repeated coding for interpretive saturation referring to the repetition of coding on different days }\end{array}$ \\
\hline Confirmability (objectivity) & $\begin{array}{l}\text { Inductive content analysis but based on the created concept map, offering modest incentive for busy } \\
\text { subjects, conscious effort at reflexivity during the interview }\end{array}$ \\
\hline $\begin{array}{l}\text { Transferability (external validity) } \\
\text { Neutrality }{ }^{19}\end{array}$ & $\begin{array}{l}\text { Sample from different clinical credentials or training backgrounds, clinics, and no other stratification } \\
\text { The sociologist Ann Oakley in } 198 \mathrm{I}^{20} \text { argued that Neutrality makes the interviewee an object and does } \\
\text { not support a cooperative engagement relationship from a social constructionism perspective. The PI } \\
\text { found that it was necessary to separate the interviewer's self-portrayal of humility from that of the } \\
\text { interviewee so that neutrality was maintained. }\end{array}$ \\
\hline
\end{tabular}


indicate a lack of dispositional humility combined with a lack of mental and emotional maturity. The data support the observation that humble clinicians would like to feel safe communicating with colleagues without feeling threatened and also to feel encouraged to approach them. Ultimately, well-functioning teams are able to develop a work culture of fairness, equality, and transparency that includes non-punitive consequences for reporting errors and near-misses.

The HH domain was also positively correlated to work productivity behaviors. ${ }^{23}$ Chirumbolo (2015) also reported that $\mathrm{HH}$ was negatively associated with the feeling of job insecurity and work counter-productivity when gender, age, types of contract, and other HEXACO traits were controlled. ${ }^{23}$ Because primary care clinicians experience a highly stressful job environment, ${ }^{17}$ dispositional humility may reduce compassion-fatigue and increase the clinician's job satisfaction. Thus, dispositional humility can be evaluated as a factor of resiliency. For all clinical disciplines (medicine, nursing, and naturopathic medicine), the high $\mathrm{HH}$ and Conscientiousness scores indicated that these clinicians were resilient to occupational stress and maintain a high degree of ethical behavior. The findings from the quantitative results were consistent with the expected results of professionals with dispositional humility. The IMAQ was created 15 years ago in $2003,{ }^{3}$ and the incorporation of integrative medicine into professional healthcare training curricula has been rapid. Furthermore, because the median years of practice of the participants in this study was 7 years, many of them had already been exposed to integrative medicine healthcare curricula in their training. As an example of this, a publication from the University of Wisconsin Integrative Medicine Elective Rotation in January 2013 lists learning objectives and defines integrative medicine as healing-oriented care for the whole person that focuses on the least invasive, least toxic, and least costly methods to help facilitate self-care, and emphasizes the personal quality of the doctor-patient relationship. ${ }^{24}$ Qualitative findings by both summative and directed content analyses extracted the descriptions, attributes, and some consequences of dispositional humility predicted by the model. The initial model created by literature search concept analysis (Figure 1) was revised (Figure 4). Professionals clearly feel that dispositional humility characteristics are important for interprofessional collaboration.

Limitations of the study include:
1. In the primary care setting, professionals from not only different modalities but also different treatment principles and health philosophies interact in the treatment of, for example, chronic health conditions. Task-specific collaborations such as in the emergency room or surgery may exhibit different teamwork dynamics. The applicability of this model of dispositional humility was examined specifically for the integrative primary care setting.

2. The opportunity for collaboration among clinicians with different training and credentials depends on geopolitical regulatory restrictions, and in this particular case, data were obtained from the state of Washington located in the United States.

3. The use of a college undergraduate sample for the reference standard of the HEXACO personality score might introduce a bias in the statistics. The age and mental maturity of individuals would have influenced dispositional factors including the $\mathrm{HH}$ score.

4. The number for the NP sample $(n=4)$ was minimal. An attempt was made to enroll 10 clinicians for each clinician type (MD/DO, NP, ND) in order to compare the dispositional factors between medicine and nursing, nursing and naturopathy, and medicine and naturopathy. This analysis was not performed due to the low number of enrollments for a particular clinician type. The number of MD/OD clinicians also did not reach the target number of ten.

5. Close inspection of the balanced sampling for the directed content analysis resulted in $3 \mathrm{MDs}, 1 \mathrm{NP}$, and 2 NDs. This unbalanced sampling of an NP replaced by an MD might have affected the abstracted data; however, we believe the effect was slight and the hypothesis of discovering the concept of dispositional humility would still be the same.

Possible future research directions are: 1) a sensitivity analysis to test the robustness of the model, for example selecting primary care clinicians at facilities which do not serve Medicaid patients; nonetheless, most community clinics that are federally qualified service Medicaid patients; 2) outcomes research on the relationship between the clinician's dispositional humility and the outcomes of patients. These outcomes are not limited to biomedical improvement of health conditions but also the tendency toward self-care and self-management of health. Because dispositional humility promotes a trusting relationship and genuine partnership, the recognition of the contribution of 
patients toward successful outcomes may influence shared responsibility in healthcare; 3) educational curricular development. The Association of Family Medicine Residency Directors describe the character traits that they look for in the National Match as: trustworthy, loyal, helpful, friendly, courteous, kind, obedient, cheerful, thrifty, brave, clean, and reverent. ${ }^{12}$ Many of these character traits are consistent with the concept of dispositional humility, and humility traits in highly determined and intelligent candidates could be facilitated by designing appropriate curricula in professional healthcare training programs; and 4) Personality tests as a tool for candidate selection. Professional identity formation considers the individual's psychological development, and the individual's recognition of their role in society and their commitment to the profession. $^{25}$ Dispositional humility, which may be reflected by the $\mathrm{HH}$ score, is useful for the candidate selection process for future/current clinicians because it may influence the clinical team dynamics as well as the clinical outcomes of patients.

\section{Conclusion}

This study demonstrated that the character trait of humility was perceived as important to facilitate productive and successful interprofessional collaboration by primary care clinicians working in a community healthcare environment. The attitude toward integrative medicine as measured by two subscales did not show a difference among clinicians with different professional healthcare training and credentials. Dispositional characteristics measured by the HEXACO showed that primary care clinicians possess statistically higher dispositional characteristics for Honesty-Humility, Conscientiousness and Openness to experience domains. The $\mathrm{HH}$ domain represents the tendency to be fair and genuine in dealing with others, while agreeableness represents tolerance and forgiveness. Non-collaborative behaviors are harmful to an organization by directly affecting its functioning or physical property, or by hurting employees. ${ }^{23}$ Interpersonal modesty refers to the tendency to moderate praise or recognition in socially acceptable ways, while humility refers to interpersonal qualities such as respect and empathy during conflict, and openness toward different cultures or worldviews; thus, humility extends the concept of modesty. ${ }^{26}$ Understanding the concept of humility is valuable for developing effective interprofessional collaborative healthcare teams. This study is a first attempt to explore the importance of dispositional humility as perceived by primary care clinicians working in community-based healthcare systems.

\section{Abbreviations}

AFMRD, The Association of Family Medicine Residency Directors; ARNP, Advanced Registered Nurse Practitioner; CAM, Complementary and Alternative Medicine; CFR, Code of Federal Regulations; DO, Doctor of Osteopathy; FISMA, Federal Information Security Modernization Act; HEXACO, Designating six domains of personality as Honesty-Humility, Emotionality, eXtraversion, Agreeableness, Conscientiousness, and Openness to experience; $\mathrm{HH}$, Honesty-Humility domain of HEXACO; HIPAA, Health Insurance Portability and Accountability Act; IMAQ, Integrative Medicine Attitude Questionnaire; MD, Medical Doctor; ND, Naturopathic Doctor; NP, Nurse Practitioner; REDCap, Research Electronic Data Capture System; USMLE, United State Medical Licensing Examination.

\section{Ethical Approval And Consent}

Bastyr University, Office of Research Integrity IRB: 16-1558. Consent for publication: signed IRB informed consent forms.

Data and materials availability: 7 years from the date of publication at Bastyr University.

\section{Acknowledgements}

Authors thank Yoriko Kozuki, Ph.D., Karen Schepp, Ph.D., and Cynthia Price, Ph.D. of the University of Washington School of Nursing. This study was self-funded.

\section{Author Contributions}

All authors contributed to data analysis, drafting or revising the article, gave final approval of the version to be published, and agree to be accountable for all aspects of the work.

\section{Disclosure}

The authors report no conflicts of interest in this work.

\section{References}

1. Sasagawa M, Amieux P. Concept map of dispositional humility among professionals in an interdisciplinary healthcare environment: qualitative synthesis. J Multidiscip Healthc. 2019;12:543-554.

2. Lee K, Ashton M. The HEXACO personality factors in the indigenous personality lexicons of English and 11 other languages. J Pers. 2008;76(5):1001-1054. doi:10.1111/jopy.2008.76.issue-5

3. Schneider CD, Meek PM, Bell IR. Development and validation of IMAQ: integrative medicine attitude questionnaire. BMC Med Educ. 2003;3:5. doi:10.1186/1472-6920-3-5

4. Eisenberg DM, Davis RB, Ettner SL, et al. Trends in alternative medicine use in the United States, 1990-1997: results of a follow-up national survey. JAMA. 1998;280(18):1569-1575. doi:10.1001/ jama.280.18.1569 
5. Loza E, Benito-Ruiz P, Blanco F, de Miguel E, Roman JA. Feasibility and efficacy of a multidisciplinary health care programme for patients with knee osteoarthritis. Clin Exp Rheumatol. 2011;29(6):913-920.

6. McDonald J, Jayasuriya R, Harris MF. The influence of power dynamics and trust on multidisciplinary collaboration: a qualitative case study of type 2 diabetes mellitus. BMC Health Serv Res. 2012;12:63. doi:10.1186/1472-6963-12-63

7. Hsieh HF, Shannon SE. Three approaches to qualitative content analysis. Qual Health Res. 2005;15(9):1277-1288. doi:10.1177/ 1049732305276687

8. Peters A, Rowatt W, Johnson M. Associations between dispositional humility and social relationship quality. Psychology. 2011;2(3):155161. doi:10.4236/psych.2011.23025

9. Rocque R, Leanza Y. A systematic review of patients' experiences in communicating with primary care physicians: intercultural encounters and a balance between vulnerability and integrity. PLoS One. 2015;10(10):e0139577. doi:10.1371/journal.pone.0139577

10. Decker SL. In 2011 nearly one-third of physicians said they would not accept new medicaid patients, but rising fees may help. Health Aff (Millwood). 2012;31(8):1673-1679. doi:10.1377/hlthaff.2012.0294

11. Decker SL. No association found between the medicaid primary care fee bump and physician-reported participation in medicaid. Health Aff (Millwood). 2018;37(7):1092-1098. doi:10.1377/hlthaff.2018.0078

12. Robinson M, Palmer E, Kozakowski S, et al. Core character traits for family medicine. Ann Fam Med. 2008;6(3):278. doi:10.1370/afm.852

13. Lee K, Ashton M The HEXACO personality inventory - revised. A measure of the six major dimensions of personality; 2015. Available from: http://hexaco.org/scaledescriptions. Accessed July 18, 2015

14. Harris PA, Taylor R, Thielke R, Payne J, Gonzalez N, Conde JG. Research electronic data capture (REDCap) - a metadata-driven methodology and workflow process for providing translational research informatics support. J Biomed Inform. 2009;42(2):377381. doi:10.1016/j.jbi.2008.08.010

15. SPSS [computer program]. Amock, NY: IBM; 2017.
16. Motulsky H The Holm-Š́lák approach to multiple comparisons; 2017. Available from: https://www.graphpad.com/guides/prism/7/sta tistics/index.htm?stat holms multiple comparison test.htm. Accessed October 1, 2019.

17. Atanes AC, Andreoni S, Hirayama MS, et al. Mindfulness, perceived stress, and subjective well-being: a correlational study in primary care health professionals. BMC Complement Altern Med. 2015;15:303. doi:10.1186/s12906-015-0823-0

18. Lincoln Y, Guba E. Naturalistic Inquiry. Newbury Park CA: Sage Publications; 1985.

19. Repley T. Interviews. In: Seale C, Gobo G, Gubrium J, Silverman D, editors. Qualitative Research Practice. London: SAGE Publications; 2007:15-33

20. Oakley A. Interviewing women: a contradiction in terms? In: Roberts H, editor. Doing Feminist Research. London: Routledge and Kegan Paul; 1981:30-61.

21. Pfattheicher S, Bohm R. Honesty-humility under threat: self-uncertainty destroys trust among the nice guys. J Pers Soc Psychol. 2018;114(1):179-194. doi:10.1037/pspp0000144

22. Ashton M, Lee K, de Vries R. The HEXACO honesty-humility, agreeableness, and emotionality factors: a review of research and theory. Pers Soc Psychol Rev. 2014;18(2):139-152. doi:10.1177/1088868314523838

23. Chirumbolo A. The impact of job insecurity on counterproductive work behaviors: the moderating role of honesty-humility personality trait. $J$ Psychol. 2015;149(6):554-569. doi:10.1080/00223980.2014.916250

24. Rakel D. The salutogenesis-oriented session: creating space and time for healing in primary care. Explore (NY). 2008;4(1):42-47. doi:10.1016/j.explore.2007.10.016

25. Chen LYC, Hubinette MM. Exploring the role of classroom-based learning in professional identity formation of family practice residents using the experiences, trajectories, and reifications framework. Med Teach. 2017;39(8):876-882. doi:10.1080/0142159X.2017.1317729

26. Davis DE, Hook JN, Worthington EL Jr., et al. Relational humility: conceptualizing and measuring humility as a personality judgment. $J$ Pers Assess. 2011;93(3):225-234. doi:10.1080/00223891.2011.558871
Journal of Multidisciplinary Healthcare

\section{Publish your work in this journal}

The Journal of Multidisciplinary Healthcare is an international, peerreviewed open-access journal that aims to represent and publish research in healthcare areas delivered by practitioners of different disciplines. This includes studies and reviews conducted by multidisciplinary teams as well as research which evaluates the results or conduct of such teams or healthcare processes in general. The journal

\section{Dovepress}

covers a very wide range of areas and welcomes submissions from practitioners at all levels, from all over the world. The manuscript management system is completely online and includes a very quick and fair peer-review system. Visit http://www.dovepress.com/testimonials. php to read real quotes from published authors. 